\title{
The recent arbovirus disease epidemic in Brazil
}

\author{
Luiz Tadeu Moraes Figueiredo ${ }^{[1]}$
}

[1]. Centro de Pesquisa em Virologia, Faculdade de Medicina de Ribeirão Preto, Universidade de São Paulo, Ribeirão Preto, São Paulo, Brasil.

In 2015, at least 9 pathogenic arboviruses that cause disease in humans have been circulating in Brazil. The Saint Louis encephalitis virus (SLEV; a flavivirus), which is transmitted from birds to humans by Culex mosquitoes, causes sporadic cases and small outbreaks of acute febrile illness and meningoencephalitis. The Mayaro virus (MAYV; an alphavirus), which is transmitted from monkeys to humans by Haemagogus mosquitoes and has a similar maintenance cycle as the sylvatic yellow fever virus) is highly endemic in the Amazon region and causes sporadic cases or outbreaks of acute febrile illness with arthropathy.

The Oropouche Orthobunyavirus (OROV; a bunyavirus) has an urban maintenance cycle in the Northern and Midwestern regions of Brazil and is transmitted human-to-human by the midge Culicoides paraensis. It has been associated with outbreaks of acute febrile illness, including some cases of meningitis. MAYV and OROV outbreaks have been recently reported in Manaus City. The majority of human cases of illness associated with these 3 arboviruses likely remain undiagnosed. In the last 3 decades, new arbovirus types have been introduced to Brazil. Dengue virus (DENV; a flavivirus) serotypes 1, 2, 3, and 4 were introduced between 1986 and 2007, mostly from Caribbean countries but in at least one case from Asia. The number of DENV outbreaks has been increasing due to the alternating predominance of the different serotypes that have been spreading across the country. During these outbreaks, approximately 10 million cases of acute febrile illness have been reported. These include dozens of thousands of patients suffering from toxic shock syndrome, meningitis, or other severe forms of the disease, resulting in thousands of deaths. The West Nile virus (WNV; another flavivirus) has likely been introduced to Brazil from North America in the past decade. West Nile virus, which is transmitted from birds to humans by Culex mosquitoes, has caused many infections in horses. The first human case of meningoencephalitis associated with WNV was reported in Northeastern Brazil (State of Piauí) in 2014. In the past

Corresponding author: Dr. Luiz Tadeu Moraes Figueiredo. Centro de Pesquisa em Virologia/FMRP/USP. Av. Bandeirantes 3900, 14049-900 Ribeirão Preto, São Paulo, Brasil.

Phone: 55 16 3602-4580; Fax: 55 16 3602-3376

e-mail: 1tmfigue@fmrp.usp.br

Received 26 May 2015

Accepted 2 June 2015
2 years, the introduction of 2 genotypes of the Chikungunya virus (CHIKV; an alphavirus), likely from the Caribbean and Asia, to different regions of the country has significantly worsened the situation. Chikungunya virus has rapidly spread through the Northern (State of Amapá), Northeastern (State of Bahia), Midwestern, and Southeastern (City of São Paulo) regions of Brazil, and outbreaks are predicted to increase in the next years. In 2015, the Zika virus (ZIKV; a flavivirus) was introduced to Brazil, likely from Asia, producing outbreaks of acute febrile illness in Northeastern Brazil (State of Bahia) and spreading to other Brazilian regions. ZIKV is transmitted to humans by Aedes spp. mosquitoes. It has recently been suggested that the virus can also be transmitted through sexual contact. Considering the recent increase in the introduction of new arbovirus types to Brazil, the question why Brazil has been experiencing this recent epidemic arises. We hypothesize that it is likely the result of a combination of factors. Infected migratory birds can travel from North to South America and thereby introduce WNV to Brazil where many Culex mosquito species, which are known to transmit the virus, are found.

The introduction of CHIKV and ZIKV is likely related to the movements of humans, in particular those associated with tourism and immigration. CHIKV would have been introduced by infected immigrants who crossed the Northern border of Brazil (State of Amapá and French Guyana). In the Northeast (State of Bahia), CHIKV might have been introduced from Asia by tourists or immigrants. The Football World Cup is an example of a large event that resulted in an increased influx of tourists from all over the world, some of whom might have been infected with an arbovirus. Finally, as has been previously reported for DENV, CHIKV and ZIKV were introduced to Brazilian urban areas through colonizing their main vector, the Aedes aegypti mosquito. This imported highly anthropophilic vector for DENV, CHIKV, and ZIKV likely allowed the fast adaptation of these viruses to our environment and resulted in the transmission to humans. We hope to have an efficient vaccine to control dengue fever in the upcoming years. For CHIKV and ZIKV, however, infection control programs rely almost exclusively on vector control due to the lack of an effective vaccine $^{(1)(2)(3)(4)(5)(6)}$.

\section{CONFLICT OF INTEREST}

The author declare that there is no conflict of interest. 


\section{REFERENCES}

1. Ministério da Saúde. Chikungunya virus in Brazil. Brasília: Ministério da Saúde; 2014.

2. Ministério da Saúde. Chikungunya and Zika virus in Brazil. Brasília: Ministério da Saúde; 2015.

3. Vasconcelos HB, Azevedo RSS, Casseb SM, Nunes-Neto JP, Chiang JO, Cantuária PC, et al. Oropouche fever epidemic in Northern Brazil: Epidemiology and molecular characterization of isolates. J Clin Virol 2009; 44:129-133.
4. Bastos MS, Figueiredo LT, Naveca F, Figueiredo R, Oliveira $\mathrm{CM}$, Gimaque JB, et al. Infection of central nervous system by Oropouche Orthobunyavirus in three Brazilian patients. Am J Trop Med Hyg 2012; 86:732-735.

5. Figueiredo MLG, Figueiredo LTM. Emerging alphaviruses in the Americas: Chikungunya and Mayaro. Rev Soc Bras Med Trop 2014; 47:677-683.

6. Figueiredo MLG, Figueiredo LTM. Review on Infections of the Central Nervous System by St Louis Encephalitis, Rocio and West Nile Flaviviruses in Brazil, 2004-2014. J Adv Microbiol 2014; 4:955-961. 